\title{
Evidence for antiseptic behaviour towards sick adult bees in honey bee colonies
}

\author{
David Baracchi ${ }^{\mathrm{a}, *}$, Antonio Fadda ${ }^{\mathrm{a}}$, Stefano Turillazzi ${ }^{\mathrm{a}, \mathrm{b}}$ \\ a Università degli Studi di Firenze, Dipartimento di Biologia Evoluzionistica “Leo Pardi”, Via Romana 17, 50125 Firenze, Italy \\ ${ }^{\mathrm{b}}$ Centro di Servizi di Spettrometria di Massa, dell'Università di Firenze, Viale G. Pieraccini, 5000 Firenze, Italy
}

\section{A R T I C L E I N F O}

\section{Article history:}

Received 16 July 2012

Received in revised form 26 September

2012

Accepted 27 September 2012

Available online 13 October 2012

\section{Keywords:}

Apis mellifera

Social immunity

Hygienic behaviour

Disease resistance

Deformed wing virus

Cuticular hydrocarbons

\begin{abstract}
A B S T R A C T
Social life is generally associated with an increased risk of disease transmission, but at the same time it allows behavioural defence at both the individual and collective level. Bees infected with deformed-wing virus were introduced into observation hives; through behavioural observations and chemical analysis of cuticular hydrocarbons from healthy and infected bees, we offer the first evidence that honeybee colonies can detect and remove infected adult bees, probably by recognising the cuticular hydrocarbon profiles of sick individuals. We also found that health-compromised colonies were less efficient at defending themselves against infected bees, thus facing an ever increasing risk of epidemics. This work reveals a new antiseptic behaviour that can only be interpreted as an adaptation at colony level and one which should be considered an element of the social immunity system of the beehive, re-enforcing the view of a colony as an integrated organism.
\end{abstract}

(c) 2012 Elsevier Ltd. All rights reserved.

\section{Introduction}

The analogy between social insect colonies and multicellular organisms was first put forward by Wheeler (1911) when insect colonies were referred to as 'superorganisms'. A century later, Strassmann and Queller (2009) proposed that a biological entity can be defined as an organism if high and nearly unanimous cooperation exists among its constituent parts, with actual conflicts among those parts largely absent or controlled. According to this view, organisms may be seen as highly cooperative social groups, and advanced eusocial insect colonies considered as organisms. Beyond the terminology, it is a biological reality that in highly eusocial insect colonies, selection may occur at both the individual and group level, while problem solving is collective in nature (Seeley, 1995; Gordon, 1996; Fewell, 2003). This evidence provides a starting point for the study of the sociality, physiology and life history of whole colonies. In superorganisms, what were once solitary organisms joined together into a cohesive whole (Bonner, 2001). Multicellularity and eusociality are accomplished by specializing individuals, cells or organisms and the development of group-level coordinating mechanisms. Multicellular organisms and insect societies face the same organizational problems and the same intense selective pressures from pathogens and parasites (Schmid-Hempel, 1998; Naug and Camazine, 2002) leading to a series of analogies in their anatomy and social anatomy, physiology and social

\footnotetext{
* Corresponding author. Tel.: +39055 2288218; fax: +39055 222565 .

E-mail address: david.baracchi@gmail.com (D. Baracchi).
}

physiology, immunity and social immunity (Cremer and Sixt, 2009; Johnson and Linksvayer, 2010).

Social life is generally associated with increased exposure to pathogens and the risk of disease transmission, due to factors such as high population density, frequent physical contact and reduced genetic variability (Alexander, 1974; Sherman et al., 1988; SchmidHempel, 1998; Côté et al., 1995; Hughes and Boomsma, 2004). On the other hand, sociality provides protection from parasites at the colony level as well as collective behavioural defence achieved by all the group members cooperating together, avoiding or eliminating parasitic infections (Cremer et al., 2007; Ugelvig and Cremer, 2007; Wilson-Rich et al., 2009) and reducing the parasite load (Rosengaus et al., 1998; Hughes et al., 2002; Traniello et al., 2002).

Like any other group of social insects, honeybee colonies, with their rich store of food, abundant mass of immature brood and adults, suffer attack from numerous pathogens and parasites (Otvos, 2000; Evans and Spivak, 2010). Moreover, the constant and relatively high temperature and humidity levels maintained in a bee nest provide the perfect environment for the incubation of all kinds of microorganisms, such as protozoa, fungi, bacteria and viruses. Like all animals, individual honeybees recruit physiological and immunological defence against disease agents (Evans et al., 2006; Schmid et al., 2008; Wilson-Rich et al., 2008). Moreover, as well as individual immunity, honeybees also show several physiological, behavioural and organizational colony-level adaptations such as spatial and behavioural compartmentalization of worker bees on the nest (Naug and Camazine, 2002; Naug, 2008), social fever (Starks et al., 2000), nest construction and enrichment 
with antimicrobial material (Simone et al., 2009; Baracchi and Turillazzi, 2010; Baracchi et al., 2011), grooming (Kolmes, 1989; Boecking and Spivak, 1999), hygienic behaviour (Rothenbuhler and Thompson, 1956; Spivak and Gilliam, 1998a,b; Richard et al., 2008), undertaking (Visscher, 1983) and self-removing (Kralj and Fuchs, 2006; Naug and Gibbs, 2009; Rueppell et al., 2010). Behaviour, in particular, plays an important role in infection control: removal, quarantine or exile of infected individuals can reduce the exposure of a population once disease takes hold (Clancy, 1996). It is well documented that many of these behaviours are commonly triggered by chemical cues. In particular, the cuticular hydrocarbons, known to play a key role in chemical communication in all social insects, mediate hygienic and undertaking behaviours in honeybees (see Evans and Spivak, 2010). Evidence exists that cuticular hydrocarbon profiles can be altered simply by activation of the immune system of bees, by using bacterial lipopolysaccharide injections (Richard et al., 2008). For example, parasitization by the Varroa destructor mite significantly alters the overall hydrocarbon profile of emerging adult bees (Salvy et al., 2001) and virusinfected honey bee workers are the target of increased grooming by nestmates (Waddington and Rothenbuhler, 1976; Drum and Rothenbuhler, 1984). However, to the best of our knowledge, although removal of corpses and hygienic behaviour towards any sick brood is pronounced in honeybees, their ability to remove health-compromised adult individuals from the nest has rarely been investigated (see Morse, 1978; Bailey, 1981 and reference within). The very first aim of this work was to verify whether honeybee (Apis mellifera) workers are able to detect bees infected with Deformed Wing Virus (DWV) and remove them from the hive soon after their emergence. Subsequently, we analysed the cuticular chemical profiles of DWV bees to see whether their hydrocarbons are modified after infection and could indeed be responsible for triggering the treatment they receive. In a second instance we also tested whether health-compromised colonies were less efficient at defending themselves against infested bees than healthy colonies, when faced with an ever increasing risk of epidemic.

\section{Materials and methods}

\subsection{Behavioural assays}

We assembled four one-frame observation hives, each consisting of about 3000 individuals plus the queen, taken from two highly deformed wing virus (DWV) infected $(\sim 1-2 \%$ of bees showing deformed wings) and two DWV free ( $\sim 0.1 \%$ of bees showing deformed wings) colonies. Bee population in the experimental hives was roughly estimated by counting the number of full or partial "sixth of frames" covered by bees in each hive at sunset (Marchetti, 1985). All the colonies were also naturally infested by the Varroa destructor mite. The comb from each colony was collected from the central part of the original hive complete with honey, pollen and brood at different stages of development.

Since healthy colonies did not provide an adequate number of newly emerged DWV bees for behavioural tests we removed healthy $(n=100)$ and DWV bees (i.e. individuals with typical crippled wing symptoms; $n=100$ ) from a single different DWV infected colony directly on their emergence from cells. Using bees from a single colony did all the tested (introduced) bees as homogeneous as possible. We marked these bees on the thorax with enamel paint and transferred them to the observation hive (25 healthy bees and 25 DWV bees for each colony: two replications were made for each type of colony, healthy and infested). To check the order of effects during the experiments, healthy and DWV bees were introduced alternatively one at a time. Each bee was observed for $10 \mathrm{~min}$ immediately after its introduction into the hive. We re- corded the number of antennations, bites and grooming events received from resident honeybees and the percentage of bees that were dragged out of the hive at the end of the census period (10 $\mathrm{min})$. The number of responses each healthy/infected bee received was normalized for the time spent on the comb (from 0 to 10 min depending on whether the bee was dragged out of the hive immediately or not during the observation period). In order to show any associations between all measured variables, PCA analysis with the dudi-mix method (a method specifically designed for PCA-type analysis on mixed categorical and continuous variables) was performed. Antennations and grooming were entered as continuous variables $(\log (x+1)$ transformed for normality and homoscedasticity) whilst bites, dragging-out- of-nest or not, bee and colony health were entered as binary factorial variables. We screened the observation hives once a day for three consecutive days counting all the marked bees. We computed and analysed survival distributions for all treatments with the Mantel-Cox Test. Healthy $(n=20)$ and DWV $(n=20)$ newly emerged bees were removed from the infected colony used for the experiment and housed in the laboratory (all the bees were kept together in a $15 \times 15 \times 15 \mathrm{~cm}$ cage in the dark, with honey and pollen ad libitum) to note their survival rate after 3 days.

\subsection{Cuticular hydrocarbon analysis}

We collected newly emerged bees from a highly infected DWV colony ( $\sim 1-2 \%$ of bees showing deformed wings), (healthy bees $n=10$; DWV bees $n=15$ ) and from a DWV-free colony ( $\sim 0.1 \%$ of bees showing deformed wings), (healthy bees $n=15$; DWV bees $n=10)$ and killed them by freezing soon after capture. Additional healthy $(n=15)$ and DWV $(n=15)$ newly emerged bees were housed in the laboratory (all the bees were kept together in a $15 \times 15 \times 15 \mathrm{~cm}$ cage in the dark, with honey and pollen ad libitum) and were killed by freezing 3 days later to check how age affected cuticular hydrocarbons. We extracted the epicuticular compounds from each bee by washing it in $200 \mu \mathrm{l}$ of hexane (with C18 hydrocarbon as internal standard) for $10 \mathrm{~min}$. The solution was then dried in a nitrogen stream and re-suspended in $25 \mu \mathrm{l}$ of heptane for GC-MS analysis. We injected $1 \mu \mathrm{l}$ of solution into a Hewlett Packard (Palo Alto, Calif.) 5890A gas chromatograph coupled with an HP 5971A mass selective detector. A fused silica capillary column coated with $5 \%$ diphenyl-95\% dimethyl polysiloxane (ZB-5.30 mm $\times 0.25 \mathrm{~mm} \times 0.1 \mu \mathrm{m}$; Zebron, USA) was used in the GC analysis. The injector port and transfer line were set at $300{ }^{\circ} \mathrm{C}$ with helium (at $12 \mathrm{psi}$ ) as the carrier gas. The temperature protocol was: $70-150{ }^{\circ} \mathrm{C}$ at a rate of $12^{\circ} \mathrm{C} / \mathrm{min}$ (held for $2 \mathrm{~min}$ ), and $150-$ $320^{\circ} \mathrm{C}$ at $8^{\circ} \mathrm{C} / \mathrm{min}$ (held for $5.08 \mathrm{~min}$ ). Analyses were performed in splitless mode. Identification of cuticular compounds was performed on the basis of their mass spectra produced by electron impact ionization $(70 \mathrm{eV})$.

We identified 39 peaks on the honeybee cuticle, each representing one or more hydrocarbons. The areas of peaks of the TIC chromatogram for each bee were normalised for the area of the internal standard (C18). To address the total CHC quantity for each bee, we summed the areas of all the peaks and compared them using the Mann-Whitney $U$ test for the newly emerged bee and 3-day-old bee groups. All the peaks were then transformed into frequencies for the sum of the peak areas considered. Before PLS-Discriminant Analysis, each peak area was transformed according to the following formula:

$Z=\operatorname{arcin} \sqrt{P}$

where $P$ is the area of the peak $i$ divided by the sum of the areas of all peaks in each specimen and $Z$ is the transformed peak area of the peak $P$. 
For analysis of hydrocarbon profiles, the resulting areas of the peaks, representing one or more cuticular compounds, were subjected to PLS-Discriminant Analysis in order to sharpen separation between the predefined groups and categories of samples (DWV bees and healthy bees) by hopefully rotating PCA (Principal Component analysis) and understanding which variables (chemical compounds) carry the class separating information. The MannWhitney $U$ test was used to compare the differences peak by peak between healthy and DWV bees.

We then calculated the Chromatographic Barycentre (CB) of the chemical profile for each bee (i.e. an imaginary point corresponding to the equilibrium of each bee's cuticular chromatogram) by replacing the mass with the percentage of each peak and the position with the respective retention time. The formula used to calculate the $\mathrm{CB}$ was:

$C B=\sum_{i=1} P i T i / \sum_{i=1} P i$

where $P i$ is the percentage area of a given compound and $T i$ is the retention time of the same compound. This method highlights the difference between the lighter and heavier compounds in a chromatogram; the central compounds are less decisive for calculating the $C B$.

Finally, to determine whether colony health influences the development of chemical profiles in honeybees, we calculated the chemical distances (using Euclidean distances and standardizing for $\mathrm{Z}$ scores) between the CHC compositions of healthy and DWV bees in the highly infested DWV colony and healthy colony, then compared them using the non-parametric Mann-Whitney $U$ test. All analyses were performed with the SPSS ${ }^{\circledR} 13.0$ statistical program and the Open source R 2.1. using the "ade4" package.

\section{Results}

\subsection{Behavioural assays}

Dudi-mix PCA extracted six PCs explaining the 100\% variance, three of which showed eigenvalues higher than 1 (Exp.Var.: PC1: 30.8\%, PC2: 17.9\%, PC3: 17.2\%). Fig. 1 shows that DWB bees have a higher probability of being bitten and dragged out of the nest than healthy ones, whilst the probability of being groomed and antennated is higher in a healthy colony than in an infested bee

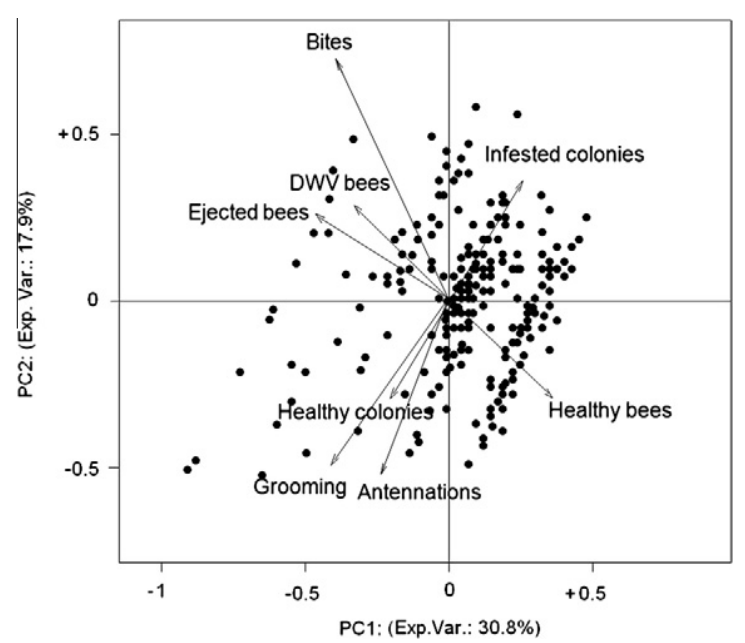

Fig. 1. Principal component analysis output with dudi-mix method. Bites $(-0.51)$ and being dragged out of the nest $(-0.58)$ are the most important variables in the PC1 (X-axis), while bites $(0.68)$ antennations $(-0.51)$ and grooming $(-0.49)$ are the most important variables in the PC2 (Y-axis).

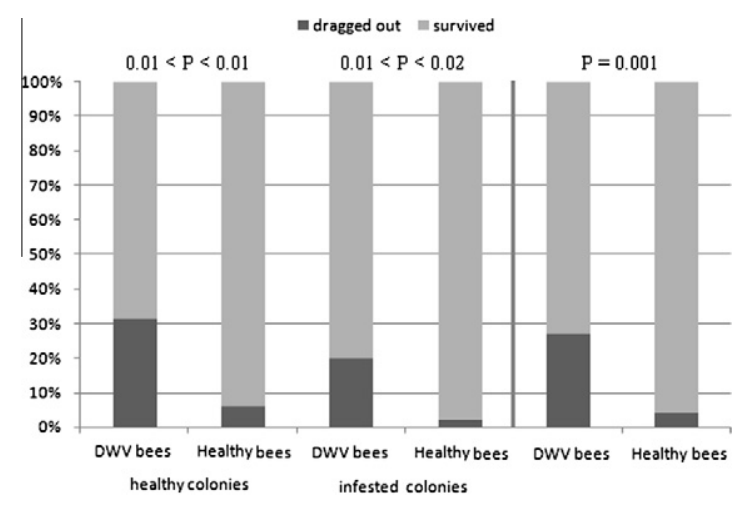

Fig. 2. Number of DWV and healthy bees dragged out of the hives $10 \mathrm{~min}$ after introduction into the colony. Results of $\chi^{2}$ test are reported for both the overall samples taken together (first and second box), and for the healthy colonies and infested colonies considered separately.

colony. After $10 \mathrm{~min}$, more DWV bees were dragged out of the hives by resident nestmates, both taking all the tested colonies together and when healthy and infested colonies were analysed separately $\left(\chi^{2}\right.$ test with Yates correction, overall: $\chi=18.47, N=200$, $\mathrm{df}=1, P<0.001$; healthy colonies: $\chi=9.27, N=100, \mathrm{df}=1,0.001$, $P<0.01$; infested colonies: $\chi=6.53, N=100, \mathrm{df}=1,0.01, P<0.02$; Fig. 2). Essentially, over the 3 days observation period, the groups of bees showed significantly different mortality rates (i.e. ejection), (Log Rank (Mantel-Cox) test, $\chi=99.34$, df =7, $P=0.0001$; Fig. 3).

Since no differences were found between the replications (Mantel -Cox test, $\mathrm{df}=1$, DWV bees in healthy colonies: $\chi=1.43, P=0.23$; DWV bee in infested colonies: $\chi=0.94, P=0.33$; healthy bees in healthy colonies: $\chi=0.001, P=0.99$; healthy bees in infested colonies: $\chi=1.31, P=0.25$; see also Fig. 3 ) the replications were clumped together. Healthy bees did not differ in ejection rate when introduced into healthy or highly infested colonies (Mantel-Cox test, $\mathrm{df}=1, \chi=0.027, P=0.87)$. In contrast, DWV bees showed higher ejection rates with respect to healthy bees, regardless of colony health (Mantel-Cox test, healthy colonies: $\chi=18.01$, $\mathrm{df}=1, \quad P<0.001$; highly infested colonies: $\chi=54.78, \quad \mathrm{df}=1$, $P<0.001$ ). Interestingly, although only two replications were made (one for highly infested and one for the healthy observation frame), the ejection rate for DWV bees changed depending on the health of the tested colonies (Mantel-Cox test, $\chi=11.93, \mathrm{df}=1, P=0.001$ ),

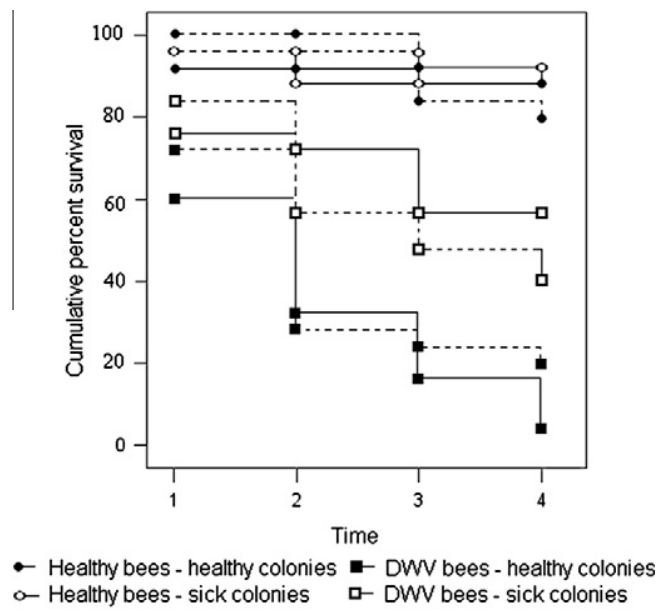

Fig. 3. Survival after introduction of healthy and DWV bees in healthy and highly infested A. mellifera colonies. Black circles: healthy bees $(N=50)$ in healthy colonies $(N=2)$; empty circles: healthy bees $(N=50)$ in sick colonies $(N=2)$; empty squares: DWV bees $(N=50)$ in healthy colonies $(N=2)$; black squares: DWV $(N=50)$ bees in sick colonies $(N=2)$. Unbroken line and dotted-line: colonies 1 and 2 . 

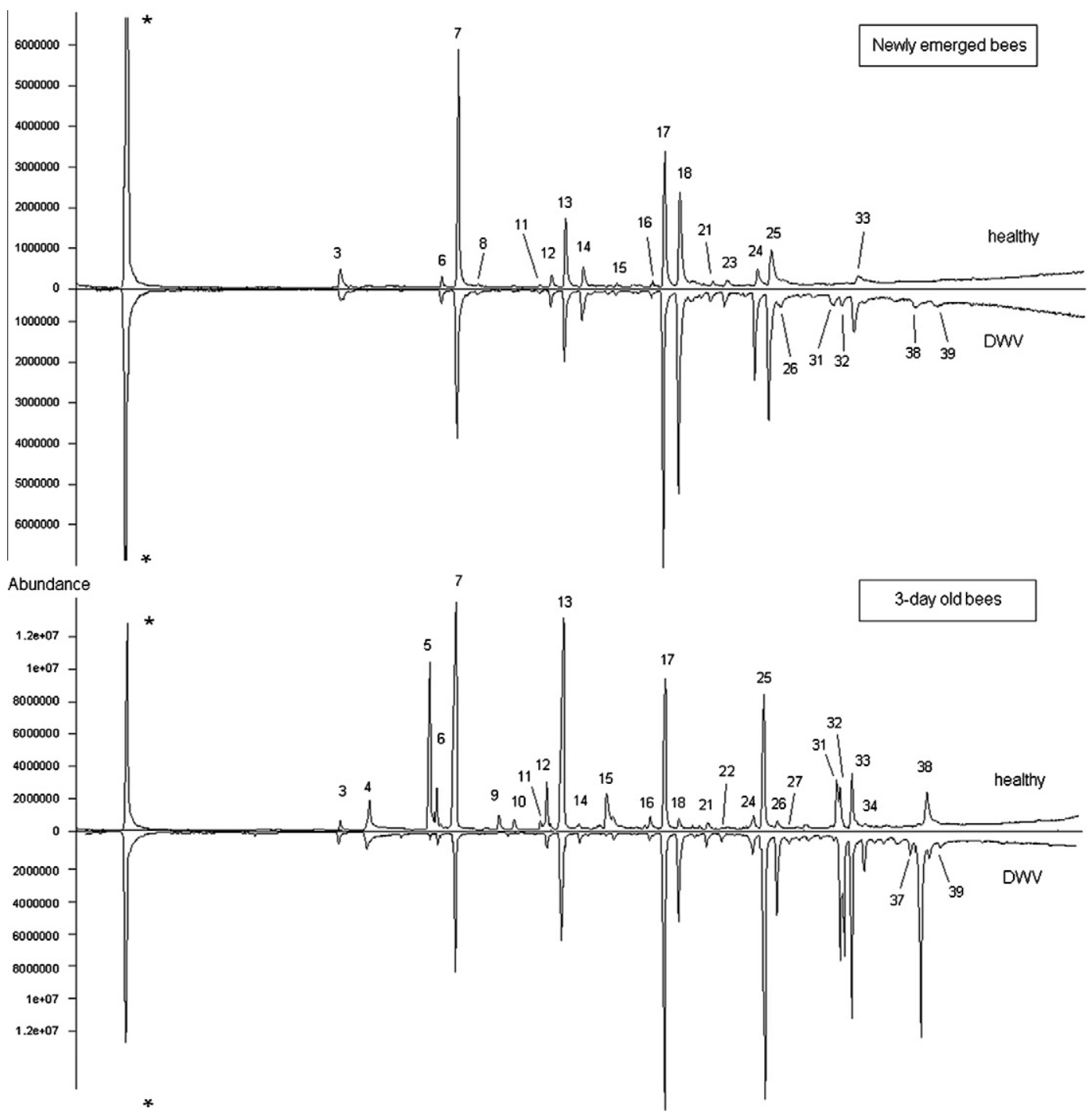

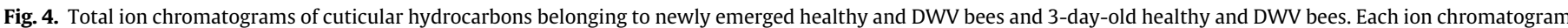

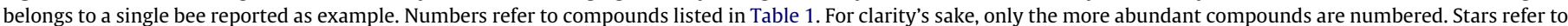
an internal standard (C18).

(Fig. 3). Finally, at the end of the three experiment days, there was no difference in mortality rate between newly emerged healthy and DWV bees reared in the laboratory ( $\chi^{2}$ test with Yates correction, $N=40, \chi=1.04, P>0.1)$.

\subsection{Cuticular hydrocarbon analysis}

We identified 39 of the compounds detected on the honeybee cuticle $(n=80)$ in the two age groups and in the different health condition groups. The cuticular waxes of bees are dominated by a series of linear and methyl-branched saturated and linear unsaturated hydrocarbons with carbon atoms numbering from 19 to 33 (Fig. 4 and Table 1). The dominant compounds were linear and mono-methyl branched compounds of odd-numbered carbon chains. Several dimethyl-branched compounds were also found in lower amounts. Unsaturated hydrocarbons were present in more variable quantities.

The total CHC amounts found on the cuticles of healthy and DWV bees were not statistically different at the time of emergence (Mann-Whitney $U$ test, $n=50, Z=-0.26, P=0.80$ ). However, as the bees grew older, $\mathrm{CHC}$ amounts increased significantly more in the healthy than DWV bees (Mann-Whitney $U$ test, $n=30, Z=-3.09$, $P<0.001$ ) (Fig. 5a).

The healthy bees had a lower Chromatographic Barycentre (newly emerged bees: median $21.56 \mathrm{~min}$, range $2.32 \mathrm{~min}$; 3-day old bees: median $22.24 \mathrm{~min}$, range $2.82 \mathrm{~min}$ ) than DWV bees (newly emerged bees: median $22.21 \mathrm{~min}$, range $1.10 \mathrm{~min}$; 3-day old bees: median $22.66 \mathrm{~min}$, range $1.13 \mathrm{~min}$ ) (newly emerged and 3-day old bees: Mann-Whitney $U$ test, $n=50, Z=-4.6, P<0.001$; $n=30, Z=-2.22, P=0.02$ respectively; Fig. 5b). Again, when newly emerged bees and 3-day old bees where grouped together, healthy bees had a lower chromatographic barycentre than DWV bees (Mann-Whitney $U$ test, $n=80, Z=-4.25, P<0.001$ ). This highlights the fact that healthy bees have a relatively greater abundance of low molecular weight compounds, while DWV bees have more higher molecular weight compounds.

Fig. 6 gives the PLS-DA classification outputs. All the bees were assigned to the correct group and most of the variance in the data set can be explained by the first function, which separates newly emerged bees from the older ones and healthy, old bees from old DWV bees, and the second function which separates newly emerged healthy bees from newly emerged DWV bees. 
Table 1

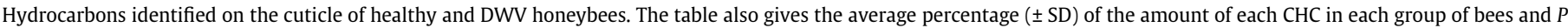
value for the Mann-Whitney $U$ test. All these substances were subsequently used in multivariate statistical analysis.

\begin{tabular}{|c|c|c|c|c|c|c|c|}
\hline Peak & Compounds & Healthy old bees & DWV old bees & $P$ value & Healthy N.E. bees & DWV N.E. bees & $P$ value \\
\hline 1 & Nonadecene & $0.59 \pm 0.03$ & $0.63 \pm 0.03$ & N.S. & $0.37 \pm 0.05$ & $0.04 \pm 0.0$ & $P=0.01$ \\
\hline 2 & Hexadecanoic acid & $2.04 \pm 0.05$ & $0.67 \pm 0.04$ & $P<0.001$ & $1.08 \pm 0.03$ & $1.14 \pm 0.06$ & N.S. \\
\hline 3 & Eneicosane & $2.89 \pm 0.03$ & $2.53 \pm 0.02$ & N.S. & $1.76 \pm 0.02$ & $2.03 \pm 0.01$ & $P=0.005$ \\
\hline 4 & Octadecenoic acid & $5.76 \pm 0.10$ & $3.10 \pm 0.09$ & $P<0.001$ & $6.98 \pm 0.08$ & $4.78 \pm 0.09$ & $P=0.003$ \\
\hline 5 & 11-Eicosen-1-ol & $0.01 \pm 0.0$ & $0.08 \pm 0.02$ & N.S. & $1.79 \pm 0.10$ & $0.31 \pm 0.03$ & N.S. \\
\hline 6 & 9-Tricosene & $2.16 \pm 0.02$ & $2.28 \pm 0.05$ & N.S. & $1.87 \pm 0.04$ & $1.75 \pm 0.03$ & N.S. \\
\hline 7 & 7-Tricosene & $1.58 \pm 0.06$ & $1.35 \pm 0.06$ & $P=0.04$ & $0.57 \pm 0.01$ & $0.96 \pm 0.0$ & $P<0.001$ \\
\hline 8 & Tricosane & $7.71 \pm 0.08$ & $6.54 \pm 0.07$ & $P=0.004$ & $5.69 \pm 0.10$ & $5.57 \pm 0.03$ & N.S. \\
\hline 9 & 9-octadecenamide & $0.25 \pm 0.02$ & $0.46 \pm 0.02$ & N.S. & $0.71 \pm 0.02$ & $0.02 \pm 0.0$ & $P<0.001$ \\
\hline 10 & Tetracosane & $1.49 \pm 0.02$ & $1.47 \pm 0.03$ & N.S. & $1.17 \pm 0.01$ & $1.07 \pm 0.0$ & N.S. \\
\hline 11 & 9-pentacosene & $1.22 \pm 0.02$ & $1.23 \pm 0.01$ & N.S. & $0.65 \pm 0.01$ & $0.88 \pm 0.01$ & N.S. \\
\hline 12 & 7-pentacosene & $2.23 \pm 0.02$ & $2.42 \pm 0.03$ & N.S. & $2.34 \pm 0.05$ & $2.11 \pm 0.01$ & N.S. \\
\hline 13 & Pentacosane & $4.82 \pm 0.03$ & $4.62 \pm 0.02$ & N.S. & $5.98 \pm 0.07$ & $4.54 \pm 0.02$ & $P=0.002$ \\
\hline 14 & 12-Methylpentacosane & $2.82 \pm 0.03$ & $2.83 \pm 0.02$ & N.S. & $1.46 \pm 0.01$ & $2.05 \pm 0.01$ & $P<0.001$ \\
\hline 15 & Hexacosane & $1.71 \pm 0.01$ & $1.65 \pm 0.01$ & N.S. & $1.69 \pm 0.01$ & $1.46 \pm 0.00$ & N.S. \\
\hline 16 & 12-Methylhexacosane & $1.52 \pm 0.01$ & $1.46 \pm 0.01$ & N.S. & $0.79 \pm 0.01$ & $1.05 \pm 0.01$ & $P<0.001$ \\
\hline 17 & 9-Heptacosene & $1.48 \pm 0.02$ & $1.80 \pm 0.08$ & N.S. & $1.54 \pm 0.01$ & $1.62 \pm 0.01$ & $P=0.015$ \\
\hline 18 & Heptacosane & $7.60 \pm 0.07$ & $8.30 \pm 0.04$ & $P=0.008$ & $8.53 \pm 0.06$ & $7.97 \pm 0.02$ & N.S. \\
\hline 19 & $11+13$-Methylheptacosane & $7.07 \pm 0.04$ & $7.15 \pm 0.06$ & N.S. & $3.28 \pm 0.05$ & $5.70 \pm 0.04$ & $P<0.001$ \\
\hline 20 & 11,x-Dimethylheptacosane & $2.17 \pm 0.05$ & $1.96 \pm 0.01$ & N.S. & $0.98 \pm 0.02$ & $1.61 \pm 0.01$ & $P<0.001$ \\
\hline 21 & Heptadecanoic a. Methyl ester & $1.32 \pm 0.01$ & $1.26 \pm 0.02$ & N.S. & $0.97 \pm 0.05$ & $1.08 \pm 0.01$ & $P=0.003$ \\
\hline 22 & Octacosane & $2.22 \pm 0.02$ & $2.45 \pm 0.01$ & $P=0.08$ & $1.80 \pm 0.02$ & $2.05 \pm 0.01$ & $P<0.001$ \\
\hline 23 & 2-Methyloctacosane & $2.81 \pm 0.03$ & $2.60 \pm 0.03$ & N.S. & $1.37 \pm 0.01$ & $2.09 \pm 0.02$ & $P<0.001$ \\
\hline 24 & 8-Nonacosene & $1.68 \pm 0.03$ & $2.09 \pm 0.04$ & N.S. & $2.51 \pm 0.02$ & $2.46 \pm 0.01$ & N.S. \\
\hline 25 & Nonacosane & $4.59 \pm 0.04$ & $5.81 \pm 0.04$ & $P<0.001$ & $6.85 \pm 0.05$ & $6.62 \pm 0.03$ & N.S. \\
\hline 26 & $11+13$-Methylnonacosane & $6.36 \pm 0.03$ & $6.49 \pm 0.05$ & N.S. & $3.07 \pm 0.05$ & $5.11 \pm 0.04$ & $P<0.001$ \\
\hline 27 & Dimethylnonacosane & $2.98 \pm 0.04$ & $2.65 \pm 0.02$ & N.S. & $1.48 \pm 0.02$ & $2.26 \pm 0.02$ & $P<0.001$ \\
\hline 28 & Triacontene & $0.63 \pm 0.04$ & $1.22 \pm 0.03$ & $P=0.029$ & $1.41 \pm 0.01$ & $1.19 \pm 0.03$ & N.S. \\
\hline 29 & Triacontane & $1.86 \pm 0.03$ & $1.79 \pm 0.03$ & N.S. & $1.32 \pm 0.01$ & $1.75 \pm 0.01$ & $P<0.001$ \\
\hline 30 & $\mathrm{x}$-Heintriacontene & $2.31 \pm 0.02$ & $2.26 \pm 0.04$ & N.S. & $2.16 \pm 0.01$ & $2.31 \pm 0.03$ & N.S. \\
\hline 31 & 8-Heintriacontene & $2.37 \pm 0.03$ & $2.72 \pm 0.03$ & N.S. & $6.02 \pm 0.05$ & $5.41 \pm 0.03$ & N.S. \\
\hline 32 & Heintriacontane & $2.26 \pm 0.04$ & $2.80 \pm 0.02$ & $P=0.01$ & $4.59 \pm 0.05$ & $4.01 \pm 0.04$ & N.S. \\
\hline 33 & Methylheintriacontane & $4.32 \pm 0.03$ & $4.32 \pm 0.03$ & N.S. & $2.10 \pm 0.03$ & $3.11 \pm 0.05$ & $P=0.001$ \\
\hline 34 & Unidentified & $2.07 \pm 0.06$ & $2.08 \pm 0.04$ & N.S. & $1.24 \pm 0.03$ & $1.53 \pm 0.04$ & N.S. \\
\hline 35 & Dotriacontene & $0.27 \pm 0.03$ & $0.27 \pm 0.03$ & N.S. & $1.65 \pm 0.01$ & $0.96 \pm 0.04$ & $P=0.002$ \\
\hline 36 & Dotriacontane & $0.15 \pm 0.02$ & $0.33 \pm 0.04$ & N.S. & $0.63 \pm 0.02$ & $0.90 \pm 0.05$ & N.S. \\
\hline 37 & Tritriacontadiene & $2.84 \pm 0.07$ & $3.24 \pm 0.04$ & N.S. & $3.77 \pm 0.06$ & $4.61 \pm 0.05$ & $P=0.003$ \\
\hline 38 & 10-Tritriacontene & $1.76 \pm 0.08$ & $2.51 \pm 0.07$ & N.S. & $5.85 \pm 0.05$ & $5.38 \pm 0.08$ & N.S. \\
\hline 39 & Tritriacontane & $0.11 \pm 0.03$ & $0.61 \pm 0.05$ & N.S. & $1.99 \pm 0.03$ & $0.75 \pm 0.06$ & $P=0.001$ \\
\hline
\end{tabular}
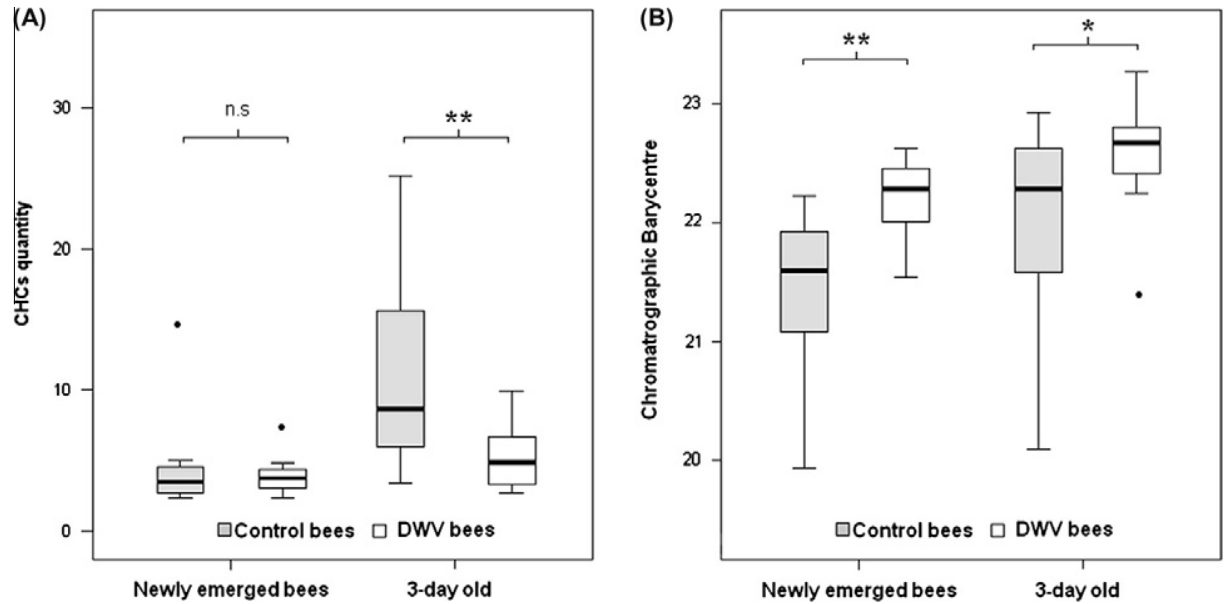

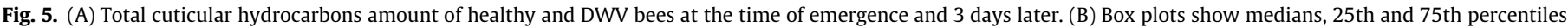
and outline data points $\left({ }^{*} P=0.02,{ }^{* *} P<0.001\right)$.

Examination of the various components contributing to differentiation between the two bee groups revealed that several compounds were important in discriminating the two bee categories. There was a mixture of saturated (C21; C24; C25; C27; C29 and C31), unsaturated ( $n-\mathrm{C} 31: 1$ and $n-\mathrm{C} 33: 2)$ and di- and mono-methyl hydrocarbons (12-meC25; $11+13-\operatorname{meC} 27 ; n$-dimeC27; 11-13-
meC29; n-dime-C29 and n-meC31), (Fig. 6). The Mann-Whitney $U$ test showed that several of these compounds differed quantitatively between healthy and DWV bees in both the newly emerged and older bees (see Table 1).

Chemical distances between healthy and DWV bees in healthy colonies did not significantly differ from those between healthy 


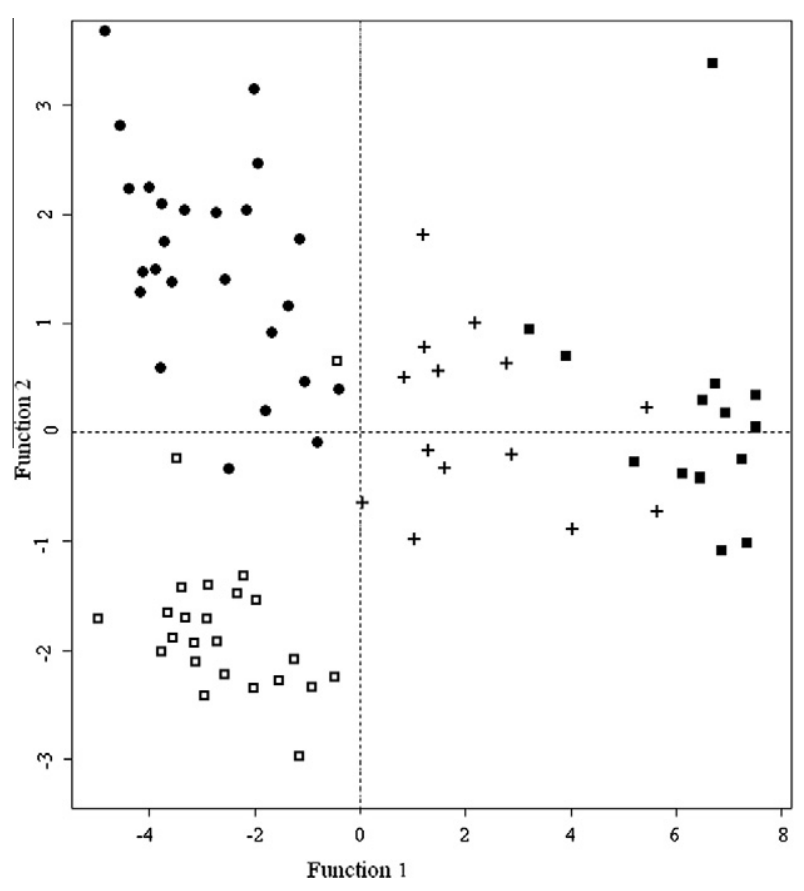

Fig. 6. PLS-Discriminant Analysis output of newly emerged healthy (black circles) and DWV bees (open squares) and 3-day-old healthy (stars) and DWV bees (black squares) using cuticular hydrocarbons as variables.

and DWV bees in highly infested colonies (Mann-Whitney $U$ test, $N=290, Z=-1.41, P=0.16)$.

\section{Discussion and conclusions}

This research furnishes the first evidence that colonies of $A$. mellifera detect and remove infected newly emerged and young adult bees from the hive. Sick individuals are an obvious threat for the colony in terms of energy costs (i.e. consumption of food resources) and increased risk of epidemics. Deformed wing virus infected bees pose a threat for the colony as: (1) quite all the $A$. mellifera colonies are naturally infested by the Varroa destructor mite and the phoretic mites are able to horizontally transmit DWV to adult healthy bees when feeding on the bees' haemolymph (Santillán-Galicia et al., 2010); (2) DWV can be transmitted horizontally to larvae via larval food containing DWV (Gisder et al., 2009; Yue and Genersch, 2005); (3) the detection of DWV in the midgut content (Fievet et al., 2006) and bee faeces (Chen et al., 2006) implies the possibility of a faecal-oral-route of transmission between adult bees. Even if handling sick individuals could increase horizontal transmission risk, there are evidence that this behaviour could be also trigger immunization of the hygenic individuals as demonstrate in termites (Traniello et al., 2002), in ants (Ugelvig and Cremer, 2007) and in bumblebee (Sadd and Schmid-Hempel, 2006). As consequence, removing sick nestmates from the colony could represent a better solution to counteract epidemics than leave them into the nest.

Several antiseptic behaviours have already been described for honey bees and insect colonies in general (Schmid-Hempel, 1998; Evans and Spivak, 2010; Hart and Ratnieks, 2001; Rosengaus et al., 1999). Hygienic behaviour (Rothenbuhler and Thompson, 1956; Spivak and Gilliam, 1998a,b) and undertaking (Visscher, 1983) are the most important behaviours which consist in detecting and removing diseased brood in the larval and pupal stages, and dragging out dead adult bees from the colony. So far, the removal of infected adult individuals has never been observed in honeybees. This behaviour is a specific form of hygienic behaviour directed towards infected adult bees rather than the infected brood, but it may also be considered similar to the entombing behaviour of termites that wall off adult nest-mates infected by nematodes (Fujii, 1975).

After the 3 days of our experiment, almost all the healthy bees but only very few infected bees were still present in the observation hives. Since both newly emerged healthy and infected bees reared in the laboratory did not differ in mortality rate, we can reasonably exclude that our results were biased by the death of sick bees. However, the sharp decrease in the survival rate of infected bees inside the hive could still be due to the combined effects of the "hygienic behaviours" carried out by resident workers as well as self-removal of infected bees. Altruistic self-removal of infected honeybee workers from their hive has been suggested by Shimanuki et al. (1994) and Kralj and Fuchs (2006), and more recently was demonstrated by Rueppell et al. (2010). Nevertheless, during the first 10 min, healthy bees were bitten and groomed significantly less than infected bees, while significantly more infected bees were dragged outside the hives. Furthermore, no DWV bees were seen crawling out of the hives, proving that, at least over a short period, the decrease in the number of infected bees in the hives was purely the effect of hygienic colony activity.

The change in cuticular hydrocarbon profiles induced by Deformed Wing Viruses infection shown by GC-MS analysis revealed a powerful discrimination between healthy bees and infected bees. These findings, together with the fact that several branchedalkanes and alkenes, compounds involved in chemical recognition (Dani et al., 2005), changing with health conditions, suggest that the CHC profiles are likely to be critical in the detection of sick individuals in beehives. Cuticular hydrocarbon profiles can be altered by activation of the immune system and their changes mediated through hygienic and undertaking behaviours in honeybees (Salvy et al., 2001; Richard et al., 2008; Evans and Spivak, 2010). No new compounds were found in the cuticular profiles of our infected bees and, according to Richard et al. (2008), our results demonstrate that neither immune stimulation by Deformed Wing Viruses nor probably by the Varroa destructor (the main factor capable of triggering visible symptoms of DWV infected bees, (de Miranda and Genersch, 2010)) produces a specific cue for disease, but rather alters the overall cuticular hydrocarbon patterns in a non-specific way. On average, $\mathrm{CHC}$ profiles of healthy bees present a relatively greater abundance of low molecular weight compounds, while the DWV bees have more higher molecular weight compounds. Furthermore, DWV bees did not show any increase in the total amount of cuticular hydrocarbons with age, suggesting that immunostimulation induced by DWV may strongly alter existing biosynthesis or transport pathways, and thereby shift cuticular chemical profiles (see also Richard et al., 2008 and references within). Interestingly, colony health did influence the immune response towards infected bees, suggesting that the development of immunity defence (i.e. social immunity defence at colony level) may depend on the social environment (i.e. the colony condition). Healthcompromised colonies were less efficient at defending themselves against infested bees, facing an ever increased risk of epidemics. Even if rejection rate may be lower in infested colonies because the DWV bee chemical profile is more similar to the overall colony gestalt odour, chemical mechanisms do not seem to be involved in this case. Indeed, there was no change in the chemical distances between healthy and infected bees in healthy or sick colonies. Possibly, in health-compromised colonies, the number of diseased bees may surpass that of hygienic bees, continuously employed in dragging out sick nestmates. On the other hand, we may hypothesize that when a colony falls sick, its social immune system and components (i.e. hygienic bees) also decline in both number and/ or quality. In any case it is still unclear whether just one or several 
factors play a role in the efficiency of detecting and removing infected nestmates; other hypotheses risk being excessively speculative. Further studies may shed some light on these issues, for example by testing DWV infected colonies with introduced bees suffering from different diseases.

In conclusion, this paper adds a new antiseptic behaviour to the already known hygienic behaviours such as allogrooming, removal of infected brood, removal of dead individuals from the nest and entombing behaviour. The most significant context for all these behaviours is presumably the prevention of disease transmission by infected brood and adult bees. The removal of infected bees can only be interpreted as an adaptation at colony level, since pathogens can quickly spread through and devastate a colony once they are established (Schmid-Hempel, 1998; Wilson-Rich et al., 2009). Hygienic bees can be considered as elements of the social immunity system of the beehive, thus strengthening the view of a colony as an integrated superorganism or an actual organism.

\section{Acknowledgements}

We thank Dr. Leonardo Dapporto of the University of Florence and Dr. Giuseppe Pieraccini of the CISM Centre for their help in chemical analysis. Many thanks to Dr. Leonardo Dapporto also for his help in statistical analysis. We also thank to Dr. Christina Coster-Longman for the revision of the English test. Finally we want to thank the anonymous referees for their useful comments and suggestions. Research was co-funded by the project APENET (DM 19735/7303/08 del 29/12/2008), and PRIN 2008 (prot. 2008KZ82RE).

\section{References}

Alexander, R.D., 1974. The evolution of social behavior. Annual Review of Ecology and Systematics 5, 325-383.

Bailey, L., 1981. Honey Bee Pathology. Academic Press, London.

Baracchi, D., Turillazzi, S., 2010. Differences in venom and cuticular peptides in individuals of Apis mellifera (Hymenoptera: Apidae) determined by MALDI-TOF MS. Journal of Insect Physiology 56, 366-375.

Baracchi, D., Francese, S., Turillazzi, S., 2011. Beyond the antipredatory defence: honey bees venom functions as a component of social immunity. Toxicon 58, 550-557.

Boecking, O., Spivak, M., 1999. Behavioral defenses of honey bees against Varroa jacobsoni Oud. Apidologie 30, 141-158.

Bonner, J.T., 2001. In: Brockmann (Ed.), First Signals: The Evolution of Multicellular Development. Princeton University Press, Princeton, NJ, p. p. 1984.

Chen, Y.P., Pettis, J.S., Collins, A., Feldlaufer, M.F., 2006. Prevalence and transmission of honeybee viruses. Applied Environmental Microbiology 72, 606-611.

Clancy, D., 1996. Carrier-borne epidemic models incorporating population mobility. Mathematical Biosciences 132, 185-204.

Côté, I.M., Poulin, R., 1995. Parasitism and group size in social mammals: a metaanalysis. Behavioural Ecology 6, 159-165.

Cremer, S., Sixt, M., 2009. Analogies in the evolution of individual and social immunity. Philosophical Transactions of the Royal Society B 364, 129-142.

Cremer, S., Armitage, S.A.O., Schmid-Hempel, P., 2007. Social immunity. Current Biology 17, 693-702.

Dani, F.R., Jones, G.R., Corsi, S., Beard, R., Pradella, D., Turillazzi, S., 2005. Nestmate recognition cues in the honeybee: differential importance of cuticular alkanes and alkenes. Chemical Senses 30, 477-489.

Drum, N.H., Rothenbuhler, W.C., 1984. Effect of temperature on non-stinging aggressive responses of worker honeybees to diseased and healthy bees. Journal of Apicultural Research 23, 82-87.

Evans, J.D., Spivak, M., 2010. Socialized medicine: individual and communal disease barriers in honey bees. Journal of Invertebrate Pathology 103, s62-s72.

Evans, J.D., Aronstein, K., Chen, Y.P., Hetru, C., Imler, J.L., Jiang, H., Kanost, M., Thompson, G.J., Zou, Z., Hultmark, D., 2006. Immune pathways and defense mechanisms in honey bees Apis mellifera. Insect Molecular Biology 15, 645-656.

Fewell, J.H., 2003. Social insect networks. Science 301 (5641), 1867-1870.

Fievet, J., Tentcheva, D., Gauthier, L., De Miranda, J.R., Cousserans, F., Colin, M.E. Bergoin, M., 2006. Localization of deformed wing virus infection in queen and drone Apis mellifera L. Virology Journal 3, 16.

Fujii, J.K., 1975. Effects of an entomogenous nematode, Neoaplectana carpocapsae Weiser, on the Formosan subterranean termite Coptotermes formosanus Shiraki, with ecological and biological studies on C. formosanus. Ph.D. Dissertation, University of Hawaii, Honolulu, Hawaii.
Gisder, S., Aumeier, P., Genersch, E., 2009. Deformed wing virus (DWV): viral load and replication in mites (Varroa destructor). Journal of General Virology 90, 463467.

Gordon, D.M., 1996. The organization of work in social insect colonies. Nature 380, $121-124$.

Hart, A.G., Ratnieks, F.L.W., 2001. Why do honey-bee (Apis mellifera) foragers transfer nectar to several receivers? Information improvement through multiple sampling in a biological system. Behavioural Ecology and Sociobiology 49, 244-250.

Hughes, W.O., Boomsma, J.J., 2004. Genetic diversity and disease resistance in leafcutting ant societies. Evolution 58, 1251-1260.

Hughes, W.O.H., Eilenberg, J., Boomsma, J.J., 2002. Trade-offs in group living: transmission and disease resistance in leaf-cutting ants. Proceedings of the Royal Society London B 269, 1811-1819.

Johnson, B.R., Linksvayer, T.A., 2010. Deconstruction the superorganism: social physiology, groundplans, and sociogenomics. Quarterly Review of Biology 85, 57-73.

Kolmes, S.A., 1989. Grooming specialists among worker honey bees, Apis mellifera. Animal Behaviour 37, 1048-1049.

Kralj, J., Fuchs, S., 2006. Parasitic Varroa destructor mites influence flight duration and homing ability of infested Apis mellifera foragers. Apidologie 37, 577-587.

de Miranda, J.R., Genersch, E., 2010. Deformed wing viruses. Journal of Invertebrate Pathology 103, s40-s61.

Marchetti, S., 1985. Il "metodo dei sesti" per la valutazione numerica degli adulti in famiglie di Apis mellifera L. Apicoltura 1, 41-61.

Morse, R.A., 1978. Honey Bee Pest Predators and Diseases. Cornell University Press, Ithaca, NY.

Naug, D., 2008. Structure of the social network and its influence on transmission dynamics in a honeybee colony. Behavioural Ecology and Sociobiology 62, 1719-1725.

Naug, D., Camazine, S., 2002. The role of colony organization on pathogen transmission in social insects. Journal of Theoretical Biology 215, 427-439.

Naug, D., Gibbs, A., 2009. Behavioral changes mediated by hunger in honeybees infected with Nosema ceranae. Apidologie 40, 595-599.

Otvos, J.D., 2000. Antibacterial peptides from insects. Journal of Peptide Science 6, 497-511.

Richard, F.J., Aubert, A., Grozinger, C., 2008. Modulation of social interactions by immune stimulation in honey bee, Apis mellifera, workers. BMC Biology 60, 50 .

Rosengaus, R.B., Maxmen, A.B., Coates, L.E., Traniello, J.F.A., 1998. Disease resistance: a benefit of sociality in the dampwood termite Zootermopsis angusticollis (Isoptera: Termopsidae). Behavioural Ecology and Sociobiology 44, 125-134.

Rosengaus, R.B., Lefebvre, M.L., Jordan, C., Traniello, J.F.A., 1999. Pathogen alarm behavior in a termite: a new form of communication in social insects. Naturwissenschaften 86, 544-548.

Rothenbuhler, W.C., Thompson, V.C., 1956. Resistance to American foulbrood in honey bees: differential survival of larvae of different genetic lines. Journal of Economic Entomology 49, 470-475.

Rueppell, O., Hayworth, M.K., Ross, N.P., 2010. Altruistic self removal of healthcompromised honey bee workers from their hive. Journal of Evolutionary Biology 23, 1538-1546.

Salvy, M., Martin, C., Bagnères, A.G., Provost, E., Roux, M., Le Conte, Y., Clement, J.L., 2001. Modifications of the cuticular hydrocarbon profile of Apis mellifera worker bees in the presence of the ectoparasitic mite Varroa jacobsoni in brood cells. Parasitology 122, 145-159.

Santillán-Galicia, M.T., Ball, B.V., Clark, S.J., Alderson, P.G., 2010. Transmission of deformed wing virus and slow paralysis virus to adult bees (Apis mellifera L.) by Varroa destructor. Journal of Apicultural Research 49, 141-148.

Schmid, M.R., Brockmann, A., Pirk, C.W.W., Stanley, D.W., Tautz, J., 2008. Adult honeybees (Apis mellifera L.) abandon hemocytic, but not phenoloxidase-based immunity. Journal of Insect Physiology 54, 439-444.

Sadd, B.M., Schmid-Hempel, P., 2006. Insect immunity shows specificity in protection upon secondary pathogen exposure. Current Biology 16, 1206-1210.

Schmid-Hempel, P., 1998. Parasites in Social Insects. Princeton Univ. Press, Princeton, NJ.

Seeley, T.D., 1995. The Wisdom of the Hive. The Social Physiology of Honey Bee Colonies. Harvard Univ. Press, Cambridge, MA.

Sherman, P.W., Seeley, T.D., Reeve, H.K., 1988. Parasites, pathogens, and polyandry in social Hymenoptera. American Naturalist 131, 602-610.

Shimanuki, H., Calderone, N.W., Knox, D.A., 1994. Parasitic mite syndrome - the symptoms. American Bee Journal 134, 827-828.

Simone, M., Evans, J.D., Spivak, M., 2009. Resin collection and social immunity in honey bees. Evolution 63, 3016-3022.

Spivak, M., Gilliam, M., 1998a. Hygienic behaviour of honey bees and its application for control of brood diseases and Varroa: Part I. Hygienic behaviour and resistance to American foulbrood. Bee World 79, 124-134.

Spivak, M., Gilliam, M., 1998b. Hygienic behaviour of honey bees and its application for control of brood diseases and Varroa: Part II. Studies on hygienic behaviour since the Rothenbuhler era. Bee World 79, 169-186.

Starks, P.T., Blackie, C.A., Thomas, D., Seeley, P.T., 2000. Fever in honeybee colonies. Naturwissenschaften 87, 229-231.

Strassmann, J.E., Queller, D.Q., 2009. The social organism: congresses, parties, and committees. Evolution 64, 605-616.

Traniello, J.F.A., Rosengaus, R.B., Savoie, K., 2002. The development of immunity in a social insect: evidence for the group facilitation of disease resistance. Proceedings of Natural Academy of Science of the United States of America 99, 6838-6842. 
Ugelvig, L.V., Cremer, S., 2007. Social prophylaxis: group interaction promotes collective immunity in ant colonies. Current Biology 17, 1967-1971.

Visscher, P., 1983. The honey bee way of death: necrophoric behaviour in Apis mellifera colonies. Animal Behaviour 31, 1070-1076.

Waddington, K., Rothenbuhler, W., 1976. Behavior associated with hairlessblack syndrome of adult honeybees. Journal of Apicultural Research 15, 35-41.

Wheeler, W.M., 1911. The ant colony as an organism. Journal of Morphology 22, 307-325.
Wilson-Rich, N., Dres, S.T., Starks, P.T., 2008. The ontogeny of immunity: development of innate immune strength in the honey bee (Apis mellifera) Journal of Insect Physiology 54, 1392-1399.

Wilson-Rich, N., Spivak, M., Fefferman, N.H., Starks, P.T., 2009. Genetic, individual, and group facilitation of disease resistance in insect societies. Annual Review of Entomology 54, 405-423.

Yue, C., Genersch, E., 2005. RT-PCR analysis of Deformed wing virus in honeybees (Apis mellifera) and mites (Varroa destructor). Journal of General Virology 86 3419-3424. 